\title{
Is HRTEM Image Simulation Correct? A Premise-Free Calibration Approach
}

\author{
Andreas Thust ${ }^{1,2}$, Juri Barthel ${ }^{1,3}$, Chun-Lin Jia ${ }^{1,2,4}$
}

${ }^{1 .}$ Ernst Ruska-Centre for Microscopy and Spectroscopy with Electrons, Forschungszentrum Jülich GmbH, 52425 Jülich, Germany.

2. Peter Grünberg Institute, Forschungszentrum Jülich GmbH, 52425 Jülich, Germany.

3. Central Facility for Electron Microscopy, RWTH Aachen University, 52074 Aachen, Germany.

${ }^{4}$ School of Electronic and Information Engineering, Xi'an Jiaotong University, Xi'an 710049, China.

The comparison of absolute image intensities between experiment and simulation in high-resolution transmission electron microscopy (HRTEM) is a long-standing problem, which led to a multitude of discussions and speculations in the past. A huge contrast mismatch, which was first stated by Hytch and Stobbs in 1994 [1], could be largely explained in 2009 as a consequence of neglecting the correct modulation transfer function (MTF) of the recording camera in image simulation [2]. Further smaller refinements to image simulation, which incorporate also the so-called image-spread effect [3], led recently to nearly perfect absolute-intensity matches between experiment and simulation $[4,5]$.

Regardless of these recent results presumptions of a huge contrast mismatch further persist [6]. Such presumptions are often based on a single remaining argument according to which a match as obtained in $[2,4,5]$ could be only a seeming one, which may have resulted from assuming a false and much too small object thickness in image simulations compared to the actual experimental thickness. The availability of a premise-free independent method for calibrating the length metrics along the beam direction, which involves the object thickness and the objective lens defocus, is therefore a valuable and clarifying goal.

We use here intentionally a radically simplified phenomenological ansatz for the image intensity $I(r, t)$ of an atomic column of height (or object thickness) $t$. The related electron wave function $\Psi(r, t)$ is formed by only two elementary mechanisms, which are (i) a single effective phase shift $\phi(r, t)$ exerted by an atomic column on the electron wave, and (ii) a subsequent propagation of the wave by a defocus $Z$. The effective phase shift is modeled as a Gaussian function $\phi(r, t)=a(t) \exp \left[-r^{2} /\left(2 w^{2}\right)\right]$ with peak value $a(t)$ and width $w$. Denoting the wavelength by $\lambda$, and omitting the thickness variable $t$ for space limitations, one obtains an analytical expression for the image intensity $I(r)$ of a defocused column in phase-object approximation, which is given here in its most compact (and unfortunately least instructive) notation:

$I(r)=\Psi(r) \Psi^{*}(r), \quad \Psi(r)=1+\sum_{n=1}^{\infty} \Psi_{n}(r), \quad \Psi_{n}(r)=\mathrm{i}^{n} \frac{2 \pi}{n n !} a^{n} w^{2} \frac{\exp \left[-\frac{\pi r^{2}}{2 \pi w^{2} / n+\mathrm{i} \lambda Z}\right]}{2 \pi w^{2} / n+\mathrm{i} \lambda Z}$

By setting $Z=0$ one recognizes that the first-order expression $\Psi=1+\Psi_{1}$ corresponds to the well-known weak-phase-object approximation. Note further that apart from the global width parameter $w$, which is nearly constant for columns of the same species, the column intensity is characterized by only two parameters, which are $a(t)$ and $Z$. Further properties which have to be known for conventional image simulations, like e.g. atomic scattering factors, Debye-Waller factors, spherical aberration, coherence properties, and camera MTF are not implemented. Above all, dynamic scattering is not correctly implemented. Our heuristic ansatz seems thus at first glance not applicable to real intensity data. 
Surprisingly it is nevertheless possible to extract meaningful $a(t)$ and $Z$ values for individual atomic columns by fitting the above expression to experimental column intensities for the following reasons: (i) Principal component analysis shows that indeed often only 2 eigenimages are needed to describe changes in thickness $t$ and defocus $Z$, which coincides with the number of the 2 parameters $a(t)$ and $Z$. Thus at least under suitable circumstances the chosen 2-parameter functionality is sufficient. (ii) The multitude of all the other rigorously discarded parameters is constant and not relevant for a distinction of varying column heights and defoci. (iii) For perfect crystalline objects $a(t)$ and $Z$ are discrete by nature. As long as nonlinear distortions introduced by the simplified approach preserve this discretization, the latter can be used for a determination of $a(t)$ and $Z$, or $t$ and $Z$, respectively, by simple level counting.

It is shown that a premise-free ab-initio calibration of object thickness and of local defocus is possible using the outlined approach when columns of light atomic species are available. The approach has been tested for an $\mathrm{MgO}$ crystal using noise-free simulated data, noisy simulated data, and experimental data. The main outcome is: (1) the results of the conventional simulation method could be perfectly confirmed and (2) the ability to perform atomic-level 3D shape reconstructions from single images, which can be achieved either with the present direct approach or with conventional simulation [5], is strongly limited by image noise and even more by an insufficient cleanliness and stability of the object surfaces.

\section{References:}

[1] MJ Hytch and WM Stobbs, Ultramicroscopy 53 (1994), p. 191.

[2] A Thust, Phys. Rev. Lett. 102 (2009), 220801.

[3] S Uhlemann et al, Phys. Rev. Lett. 111 (2013), 046101.

[4] CL Jia et al, Microsc. Microanal. 19 (2013), p. 310.

[5] CL Jia et al, Nature Materials 13 (2014), p. 1044.

[6] D Van Dyck et al, Micron 68 (2014), p. 158.

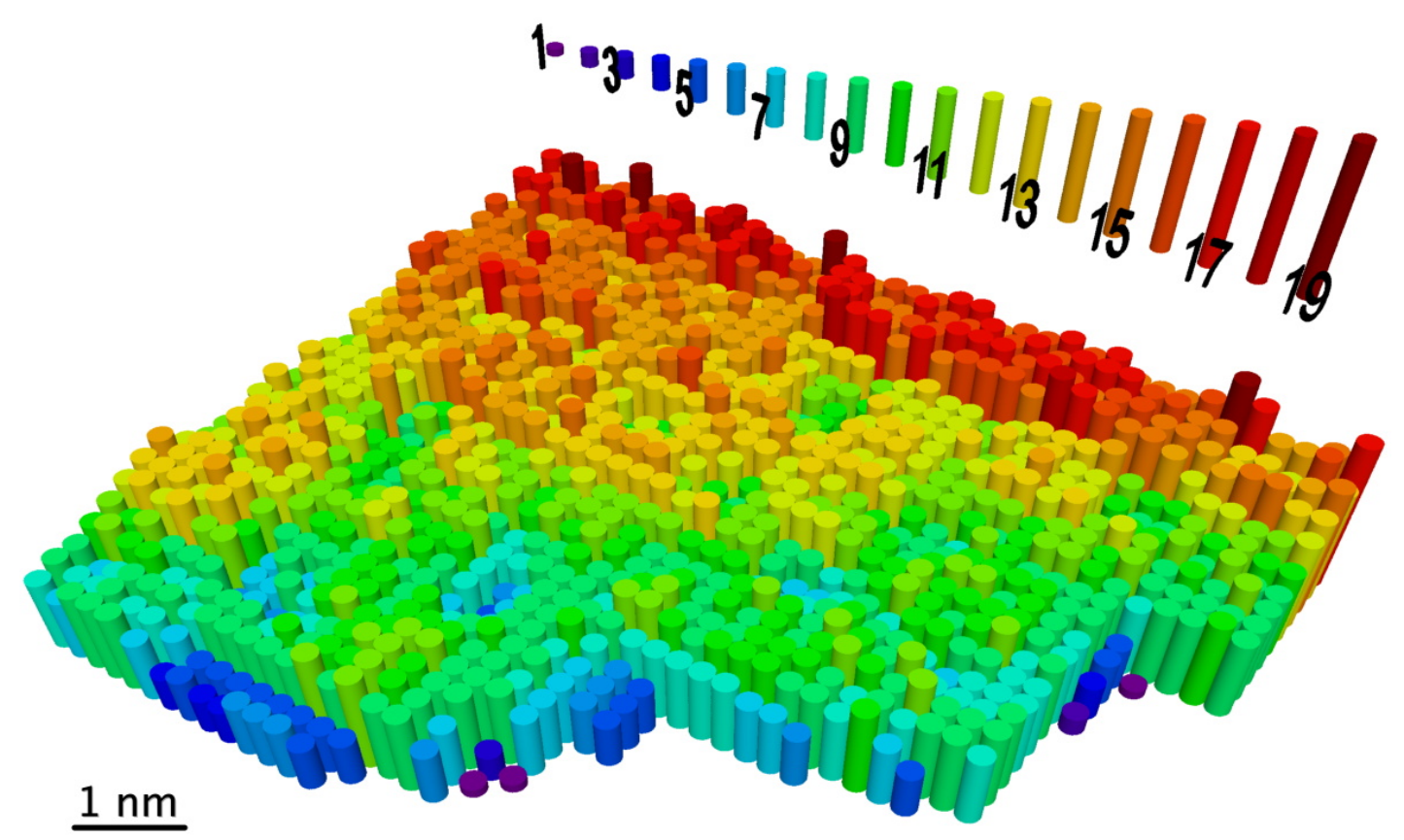

Figure 1. Thickness map extracted from a single experimental HRTEM image of an $\mathrm{MgO}$ crystal edge taken along the [001] zone axis. The discrete height of the atomic columns reaches from 1 to 19 atoms. 